\title{
Activation of the Toll-like receptor 8 pathway increases the immunogenicity of mesenchymal stem cells from umbilical cord
}

\author{
YU YANG ${ }^{1}$, YANWEN WANG $^{2}, \mathrm{LI} \mathrm{LI}^{2}$, FEI CHEN ${ }^{2}$ and PENG ZHANG ${ }^{3}$ \\ ${ }^{1}$ Department of Medical Oncology, Cancer Center; ${ }^{2}$ Laboratory of Pathology, Department of Pathology, \\ ${ }^{3}$ Department of Urology, West China Hospital, Sichuan University, Chengdu, Sichuan 610041, P.R. China
}

Received April 25, 2016; Accepted March 21, 2017

DOI: $10.3892 / \mathrm{mmr} .2017 .6806$

\begin{abstract}
Mesenchymal stem cells (MSCs) are now widely used in clinical cell-based therapy due to their characteristics of low immunogenicity, multiple differentiation potency and the capability to modulate immune responses. However, accumulated research has indicated the absence of engrafted MSCs because of the increased immunogenicity of MSCs. Toll-like receptors (TLRs) are essential for the innate immune response and regulating the biological function of MSCs. The present study used human umbilical cord-derived MSCs (UCMSCs) and activated the TLR8 pathway of UCMSCs to study the role of TLR8 in mediating the immune status of UCMSCs. The results demonstrated that the activation of TLR8 increased both the proliferation of peripheral blood mononuclear cells (PBMCs) isolated from healthy human volunteers and the release of lactate dehydrogenase (LDH) in supernatant from the PBMC-UCMSCs co-culture system. Reverse transcription-quantitative polymerase chain reaction indicated that the TLR8 agonist increased the expression of many co-stimulatory molecules and pro-inflammatory genes, and flow cytometry indicated that activation of the TLR8 agonist increased co-stimulation protein levels but reduced specific surface markers, as confirmed by the part loss of stemness of UCMSCs. Finally, TLR8 increased osteocyte differentiation but had no effect on chondrocyte and adipocyte differentiation. The current study indicated the implication to TLR8 as regulators of the immunogenicity of UCMSCs.
\end{abstract}

\section{Introduction}

Mesenchymal stem cells (MSCs) can be derived from various tissues, including fat, muscle and the umbilical cord. MSCs are

Correspondence to: Professor Peng Zhang, Department of Urology, West China Hospital, Sichuan University, 37 Guo Xue Xiang, Chengdu, Sichuan 610041, P.R. China

E-mail: zpeng2001@163.com

Key words: umbilical cord mesenchymal stem cells, toll-like receptor 8, immunogenicity, pro-inflammatory molecules, co-stimulation factors capable of self-renewing and differentiating into distinct cell types, such as chondrocytes, osteoblasts and adipocytes, and thus hold promise for regenerative medicine (1). MSCs express several surface proteins, including CD29, CD59, CD90, CD105 and CD44, but low levels of undetectable hematopoietic markers, such as CD14, CD34, CD31 and HLA class I molecules (2). MSCs also lack expression of co-stimulatory CD80, CD86 or CD40, even following IFN- $\gamma$ stimulation (3). Another important feature of MSCs is that they may inhibit activation, proliferation and function of immune cells, including $\mathrm{T}$ cells, B cells, NK cells and antigen-presenting cells (4-6). Due to these features, MSCs hold great promise for treating various diseases. MSCs had now been used in many clinical trials to treat many diseases, such as graft vs. host disease, live fibrosis and cardiovascular diseases (5-7). However, accumulated evidence indicates that engrafted MSCs could stimulate the immune responses and, finally, result in the rejection of MSCs (8). In murine bone marrow transplantation models, memory T cells may reject the engrafted MSCs (9) and it was also demonstrated that NK cell ligands, which expressed on MSC, mediated the rejection of MSC by NK cells (10).

The Toll-like receptor (TLR) family involves types of molecules that are expressed in immune and non-immune cells and act as important sensors for the detection of heterogeneous pathogens and endogenous danger signals (11). Moreover, generally TLRs activate a rapid effector response, which implies intrinsic and adaptive immune responses (12). Stimulation of cells with a TLR ligand recruits intracellular adaptor proteins, including myeloid-differentiation primary-response protein 88 and adaptor-inducing interferon (IFN)- $\beta$, triggering downstream signaling cascades and productions of pro-inflammatory cytokines and chemokines (13). A previous study indicated that MSCs are activated by TLR ligands leading to modulation of the differentiation, migration, proliferation, survival and immunosuppression capacities (14). TLR3 in MSCs has been reported to significantly prolong the survival and function of neutrophils (14). It was also reported that TLR8 and TLR9 activation increased the migration of MSCs, but activation of the TLR8 pathway had no significant effects on immunogenicity, immunosuppression and survival of MSCs when isolated from bone marrow and adipose (15-17).

In the present study, MSCs were isolated from the umbilical cord and stimulated with the TLR8 agonist, R848. The 
aim of the current study was to evaluate the role of TLR8 in mediating the changing of immune status of UCMSCs.

\section{Materials and methods}

Isolation and culture of UCMSCs. The UCMSCs were provided by the Sichuan Cord Blood Bank (Chengdu, China) and maintained in Dulbecco's modified Eagle's medium (Invitrogen; Thermo Fisher Scientific, Inc., Waltham, MA, USA) containing $10 \%$ fetal bovine serum, $2 \mathrm{mM}$ glutamine, $1 \mathrm{mM}$ sodium pyruvate, $100 \mathrm{U} / \mathrm{ml}$ penicillin and $100 \mathrm{~g} / \mathrm{ml}$ streptomycin (all from Invitrogen; Thermo Fisher Scientific, Inc.), and subcultured every 3-4 days by using fresh media. UCMSCs were incubated at $37^{\circ} \mathrm{C}$ in a $5 \% \mathrm{CO}_{2}$ humidified atmosphere and used in the experiments only after 2 to 3 expansion passages to ensure depletion of monocytes/macrophages.

TLR8 agonists that stimulate MSCs. TLR8 reagent R848 was from Enzo Life Sciences, Inc. (ALX-420-040; Farmingdale, NY, USA) and dissolved in DMSO as a storage concentration of $25 \mathrm{mg} / \mathrm{ml}$. UCMSCs were seeded into six-well plate at a concentration of $1.5 \times 10^{5}$ in $2 \mathrm{ml}$ medium and the TLR8 agonist was added into the UCMSC culture medium at a final stimulation concentration of $5 \mu \mathrm{g} / \mathrm{ml}$. PBMCs were marked with carboxyfluorescein succinimidyl ester.

Reverse transcription-quantitative polymerase chain reaction (RT-qPCR). Total RNA was isolated from confluent MSCs using the RNeasy mini kit (74104; Qiagen GmbH, Hilden, Germany). cDNA synthesis was performed using ReverTra Ace qPCR RT kit (FSQ-101; Toyobo Co., Ltd., Osaka, Japan) under the conditions: $65^{\circ} \mathrm{C}$ for $5 \mathrm{~min}$, followed by $37^{\circ} \mathrm{C}$ for $15 \mathrm{~min}$ and $98^{\circ} \mathrm{C}$ for $5 \mathrm{~min}$. The levels of mRNA of genes of interest were measured by RT-qPCR (iCycleriQ ${ }^{\text {Tw }}$ Optical Module, Bio-Rad Laboratories, Inc., Hercules, CA, USA) using RealMaster Mix (SYBR-Green; FP202, Tiangen Biotech Co., Ltd., Beijing, China), under the following conditions: One cycle at $95^{\circ} \mathrm{C}$ for $30 \mathrm{sec}, 40$ cycles at $95^{\circ} \mathrm{C}$ for $30 \mathrm{sec}, 58^{\circ} \mathrm{C}$ for $30 \mathrm{sec}$ and $72^{\circ} \mathrm{C}$ for $30 \mathrm{sec}$, followed by a melt curve from 55 to $95^{\circ} \mathrm{C}$ in $0.5^{\circ} \mathrm{C}$ increments and $10 \mathrm{sec}$ intervals.

Total amount of mRNA was normalized to endogenous GAPDH mRNA (18). Sequences of PCR primer pairs are presented in Table I. All experiments were performed three times.

Antibody chip array. Analyzed secretion of protein of supernatant from two groups TLR8-treated and untreated UCMSCs were collected $4 \mathrm{~h}$ post stimulation using the RayBio Human Antibody Array C Series 1000 (RayBiotech, Inc., Norcross, GA, USA) according to the manufacturer's protocols. Blots were analyzed with ImageJ software version 2.0 (National Institutes of Health, Bethesda, MD, USA).

Flow cytometry analysis. For flow cytometry analysis, the following monoclonal antibodies for detection of co-stimulator and surface markers were used: CD80, CD86, HLA-E, CD90, CD59, CD29, CD80, CD86 and HLA-1 were used for co-stimulator detection while CD90, CD59 and CD29 were surface markers of MSC (Table II). TLR8-treated and untreated
UCSMCs were harvested $72 \mathrm{~h}$ post stimulation and incubated with the monoclonal antibodies for $30 \mathrm{~min}$ then washed twice with phosphate buffered saline, and appropriate isotopic controls were included. Flow cytometry was performed using a FACScan flow cytometer (BD Biosciences, Franklin Lakes, NJ, USA). The acquisition and analysis gates were chosen based on the high fluorescence intensity of control group.

UCMSC differentiation assay. To assay UCMSC differentiation, conditioned medium specific was added to chondrogenesis (A10071-01), osteogenesis (A10072-01) and adipogenesis (A10070-01) differentiation kits (all from Gibco; Thermo Fisher Scientific, Inc.). UCMSCs were inoculated into a six-well plate $\left(1.5 \times 10^{5}\right)$ containing $2 \mathrm{ml}$ differentiation medium, TLR8 agonist was added at a concentration of $5 \mu \mathrm{g} / \mathrm{ml}$. The conditioned mediums were replaced every 3 days. UCMSCs both treated and untreated by TLR 8 were stained for 5, 10 and 15 days for different cell types. In this assay, Oil Red $\mathrm{O}$ was used for staining of adipocytes, Alizarin Red for osteocytes and Safranin for chondrocytes.

Statistical analysis. Data are presented as mean \pm standard deviation and performed by SPSS software version 16.0 (SPSS Inc., Chicago, IL, USA). Statistical significance was assessed by unpaired two-tailed Student's t-test and differences were $\mathrm{P}<0.05$ was considered to indicate a statistically significance difference. GraphPad Prism software version 5 (GraphPad Software, Inc., La Jolla, CA, USA) was used for diagrams.

\section{Results}

Activation of TLR8 significantly increases the proliferation of peripheral blood mononuclear cells (PBMCs) and leakage of $L D H$. To assess whether TLR8 agonist R848 increased the proliferation of PBMCs, PBMCs were marked with carboxyfluorescein succinimidyl ester and co-cultured with UCMSCs with or without TLR8 activation. The PBMCs were isolated from healthy human volunteers and then co-cultured with UCMSCs in the presence of R848. The results indicated that PBMCs proliferated dramatically in the PBMCs-UCMSCs-R848 group (24.8\%) following $72 \mathrm{~h}$ co-culture compared with control groups (PBMC-MSC, 9.2\%, PBM-Neg, 3.1; Fig. 1A). The result confirmed the induced immune response upon TLR8 activation.

The next detection was performed to detect leukocyte-mediated cytotoxicity by measuring the LDH levels in supernatant released by damaged cells. In the assay, the supernatant from the PBMC-MSC co-culture was collected for detection of LDH levels. The LDH levels were demonstrated as not obviously different at 24 and $48 \mathrm{~h}$ co-culture in the presence of the TLR8 agonist. However, the LDH level was significantly increased in the TLR8 treatment group when compared with non-treatment group $(\mathrm{P}<0.05$; Fig. 1B). The LDH detection indicated that activation of TLR8 increased the damage of UCMSCs.

TLR8 ligand dramatically increases the expression of pro-inflammatory cytokines. UCMSCs were stimulated by specific ligand R848 and then the expression variation of tumor-related genes (CDC2, PTEN, PCNA and VEGF), 
Table I. List of oligonucleotides used for reverse transcription-quantitative polymerase chain reaction analysis.

\begin{tabular}{llll}
\hline Gene number & \multicolumn{1}{c}{ Forward primer } & \multicolumn{1}{c}{ Reverse primer } & \multicolumn{1}{c}{ GenBank } \\
\hline CDC2 & CAGGTTATATCTCATCTTTGAG & GTTGAGTAACGAGCTGACCCC & AM393287 \\
PTEN & ACCATAACCCACCACAGC & CAGTTCGTCCCTTTCCAG & NM_058074 \\
PCNA & ATTCCAGAACAGGAGTACAGCTGT & CAGATGTACCCCTTGTTGAGAGT & NM_002592 \\
VEGF & GACTTGAGTTGGGAGGGAA & GAGGCTCAGCGCCAGGGCTGGG & AF024710 \\
CD80 & TGGCAACGCTGTCCTGTG & CCTTTTGCAGTAGATGCGAG & M27533 \\
CD86 & GGGCCGCACAAGTTTTGA & GCCCTTGTCCTTGATCTGAAGA & L25259 \\
IL-1 $\beta$ & ACGAATCTCCGACCACCACT & CCATGGCCACAACAACTGAC & M15330 \\
IL-6 & GACCCAACCACAAATGCCA & GTCATGTCCTGCAGCCACTG & M14584 \\
IL-8 & CTGGCCGTGGCTCTCTTG & CCTTGGCAAAACTGCACCTT & NM_000584 \\
IL-9 & CTCTGTTTGGGCATTCCTCT & GGGTATCTTGTTTGCATGGTGG & M30134 \\
IL-10 & GGTGATGCCCCAAGCTGA & TCCCCCAGGGAGTTCACA & U16720 \\
IL-1 1 & CGAGCGGACCTACTGTCCTA & GCCCAGTCAAGTGTCAGGT & NM_000641 \\
IL-12 & CGGTCATCTGCCGCAAA & CAAGATGAGCTATAGTAGCGGTCCT & M65272 \\
IL-15 & TTTCTAACTGAAGCTGGCATTCAT & CCAGTTGGCTTCTGTTTAGGA & U14407 \\
I-309 & GCAGATCATCACCACAGCC & GTCCACATCTTCCGGCCA & NM_002981 \\
EOTA-3 & CCAAGACCTGCTGCTTCCAA & GAATTCATAGCTTCGCACCCA & NM_006072 \\
MCP-4 & CAGTGCTTCTGTGCCTGCTG & TGCATCTGGCTGAGCAAGTC & NM_005408 \\
MIP-1 $\beta$ & CTGCTCTCCAGCGCTCTCA & GTAAGAAAAGCAGCAGGCGG & NM_002984 \\
GAPDH & GAAGGTGAAGGTCGGAGTC & GAAGATGGTGATGGGATTTC & J04038 \\
\hline
\end{tabular}

Table II. Monoclonal antibodies for fluorescence activated cell sorting analysis.

\begin{tabular}{llc}
\hline Name & Company & Catalog number \\
\hline CD80 & eBioscience & $11-0809$ \\
CD86 & eBioscience & $12-0869$ \\
HLA-E & eBioscience & $17-9953$ \\
CD90 & eBioscience & $45-0909$ \\
CD59 & eBioscience & $11-0596$ \\
CD29 & eBioscience & $17-0299$ \\
\hline
\end{tabular}

eBioscience, Inc., San Diego, CA, USA.

co-stimulators (CD80, CD86), cytokines (IL-1 $\beta,-8,-9,-10$, $-11,-15)$ and chemokines (I309, EOTA-3, MCP-4 and MIP-1 $\beta$ ) were measured. The TLR8-treated UCMSC mRNAs were isolated and detected on different time points $(4,12,24,72$ and $120 \mathrm{~h}$ ) and assayed by RT-qPCR.

The fold induction of CDC2 production in response to R848 increased greatly and reached the peak at $4 \mathrm{~h}$ then gradually decreased. The response study indicated that TLR8 ligand activation induced production of PTEN, PCNA and VEGF. While expression of PCNA increased 4-, 12- and $24 \mathrm{~h}$ post stimulation but decreased from 72- and $120 \mathrm{~h}$ (Fig. 2A). Co-stimulators CD80 and CD86 presented a noticeable rise following stimulation by TLR8 ligand (Fig. 2B). Interleukins IL- $\beta,-9,-10,-11$ and -15 expression increased upon TLR8 agonist stimulation, but IL-8 decreased, even under the level of the negative control at $120 \mathrm{~h}$ (Fig. 2B). With chemokines I309, EOTA-3, MCP-4 and MIP-1 $\beta$, the data demonstrated that the fold induction of chemokines production in response to R848 increased greatly and EOTA-3, MIP-1 $\beta$ reached a peak at $24 \mathrm{~h}$, whilst MCP-4 reached a peak at $72 \mathrm{~h}$ (Fig. 2C).

TLR8 agonist induces the expression of co-stimulatory molecules and inhibited the expression of stem makers in UCMSCs. Gene expression and UCMSCs-PBMC co-culture observations suggested that the TLR8 agonist greatly increased the immunogenic response. It was hypothesized that TLR8 may upregulate the expression of co-stimulation molecules, which finally mediated the TLR8-induced immune response. To analyze co-stimulation protein expression in UCMSCs, flow cytometry was used with specific antibodies to human CD86, CD80 and HLA-E. Negative control UCMSCs and TLR8-treated UCMSCs were collected 5 days post-stimulation. Flow cytometry analysis indicated that three co-stimulation proteins had increased in various degrees by TLR8 treatment, the most notable being CD80 (39\% vs. 2.1\%; Fig. 3A). In addition, specific surface MSC markers CD29, CD90 and CD59 were used for detection. TLR8 treatment of UCMSCs resulted in the decreased expression of CD29 (83.6\% vs. $95.1 \%)$ and $\operatorname{CD} 59$ (81.8\% vs. $97.5 \%)$, which suggested that stemness of UCMSCs was diminished upon TLR8 agonist treatment (Fig. 3B).

TLR8 stimulation in UCMSCs induces the secretion of cytokines. To assess whether TLR8 can enhance the cytokine secretion of UCMSCs, an antibody chip assay was performed to detect the dose of cytokines. UCMSCs were stimulated with the TLR8 ligand for $4 \mathrm{~h}$ prior to supernatant collection and 20 cytokines, chemokines and other important factors were analyzed (AFP, albumin, E-selectin, ICAM-1, IFN- $\alpha$, IFN- $\gamma$, IL-10, IL-12, IL-18, IL-1 $\beta$, IL-4, IL-5, IL-6, 
A
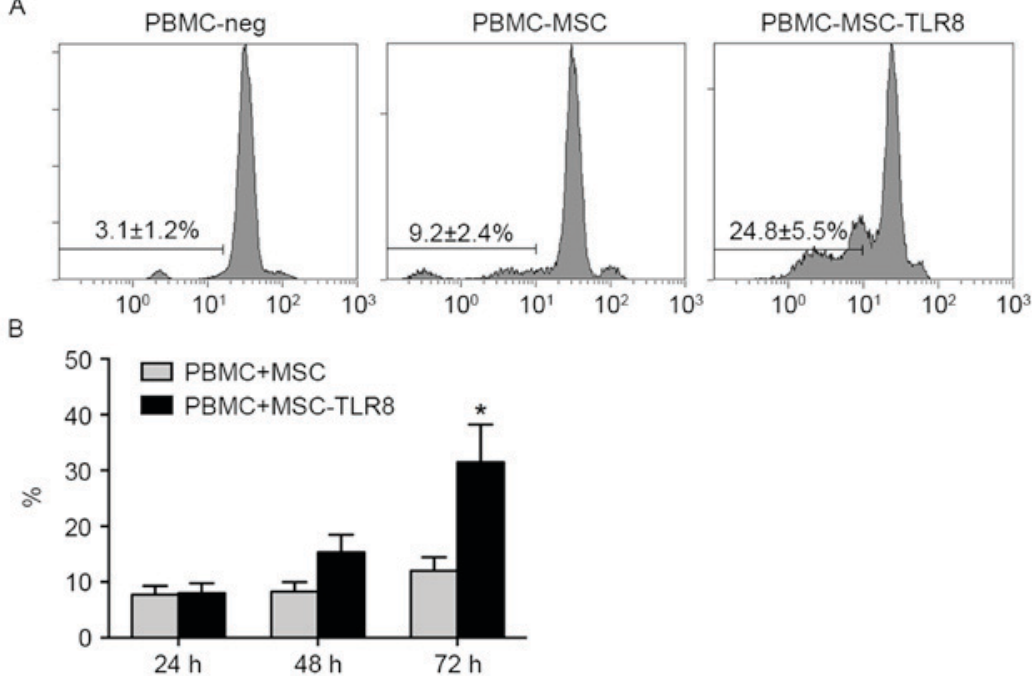

Figure 1. Proliferation of PBMCs and cytotoxicity detection. (A) Co-culture of PBMCs and UCMSCs. (B) Lactate dehydrogenase detection for cytotoxicity. "P<0.05 vs. PBMC + MSC. PBMC, peripheral blood mononuclear cell; MSC, mesenchymal stem cell; UBMSC, umbilical cord-derived mesenchymal stem cell.

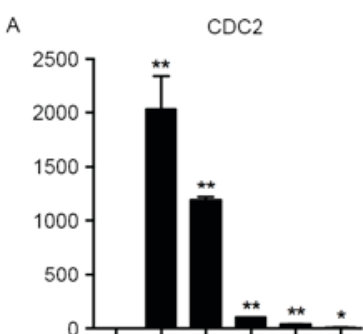

CD80
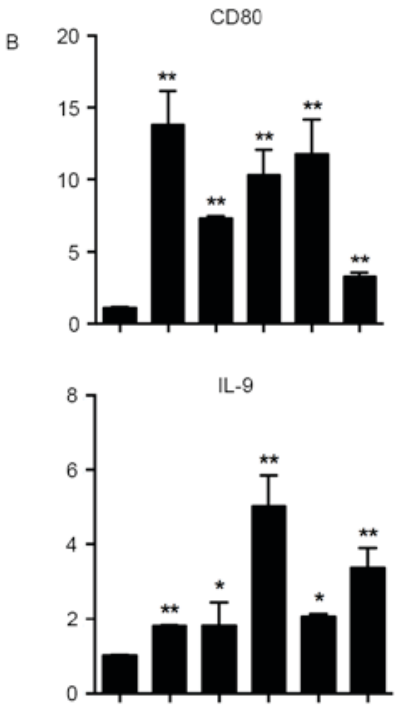

1309

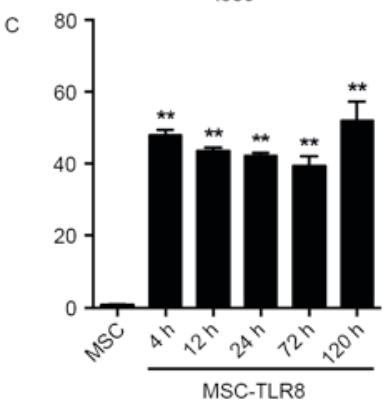

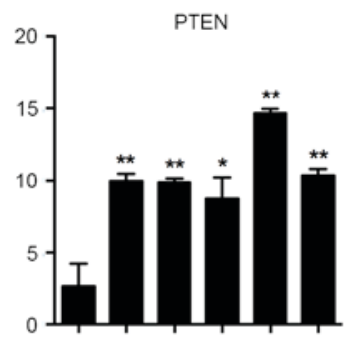

CD86

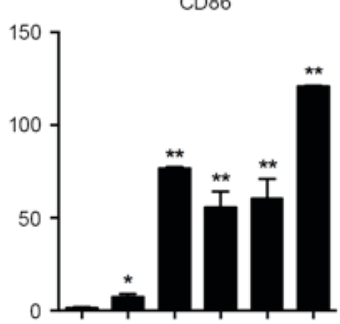

$\mathrm{IL}-10$
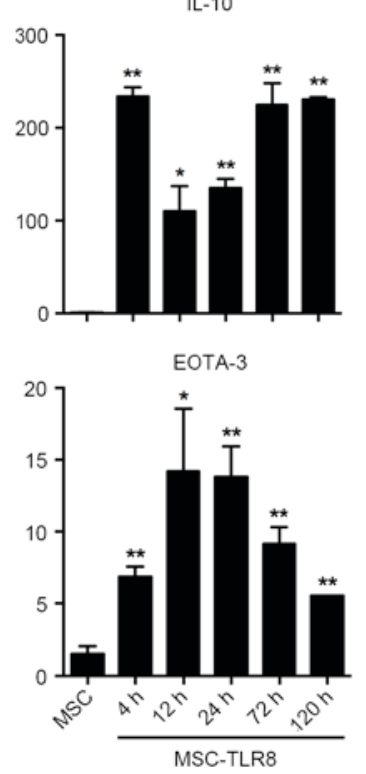
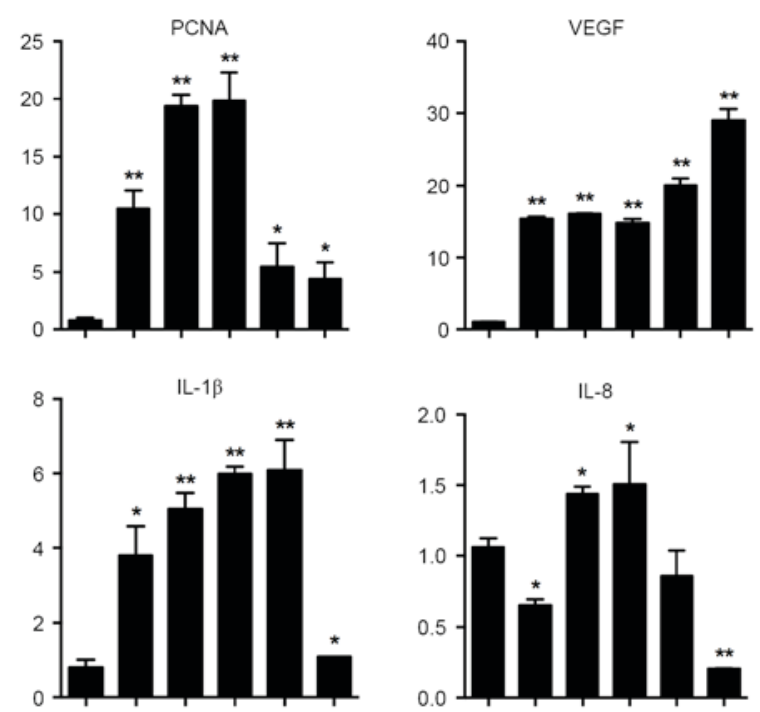

IL-11

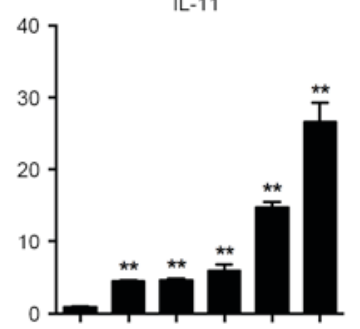

MCP-4

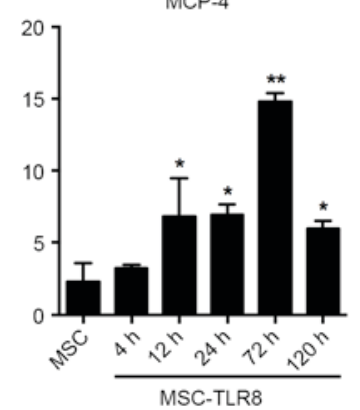

IL-15

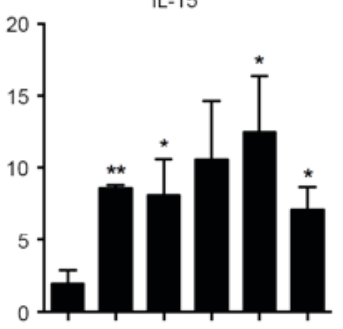

MIP-1 $\beta$

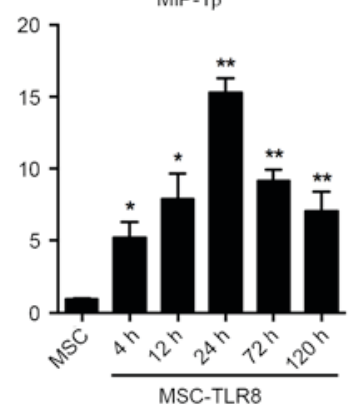

Figure 2. Gene expression variation detection of (A) tumor-related genes, (B) co-stimulators and interleukins and (C) chemokines. MSC group, untreated MSC; MSC-TLR8, TLR8 agonist-treated MSCs. ${ }^{*} \mathrm{P}<0.05,{ }^{* *} \mathrm{P}<0.001$ vs. MSC group. MSC, mesenchymal stem cell; TLR-8, Toll-like receptor 8 . 
A
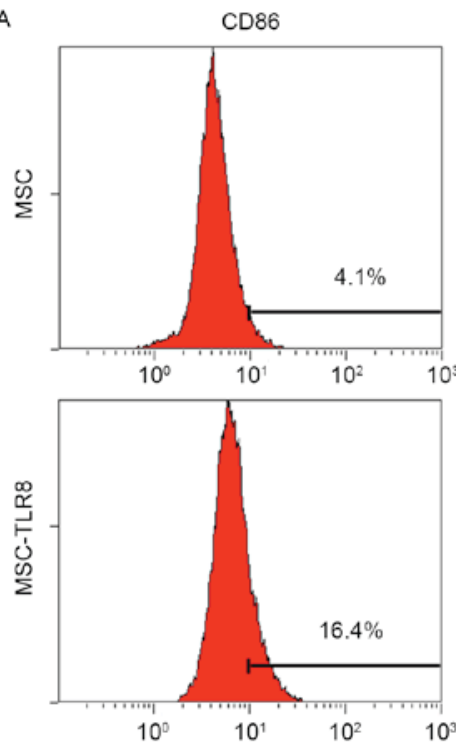

B
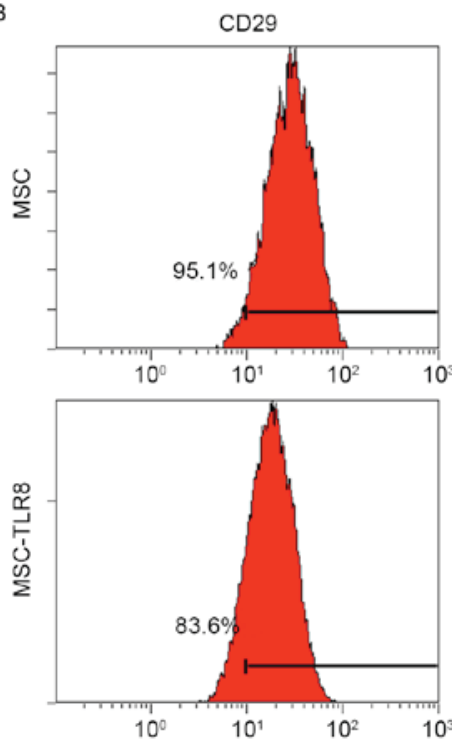
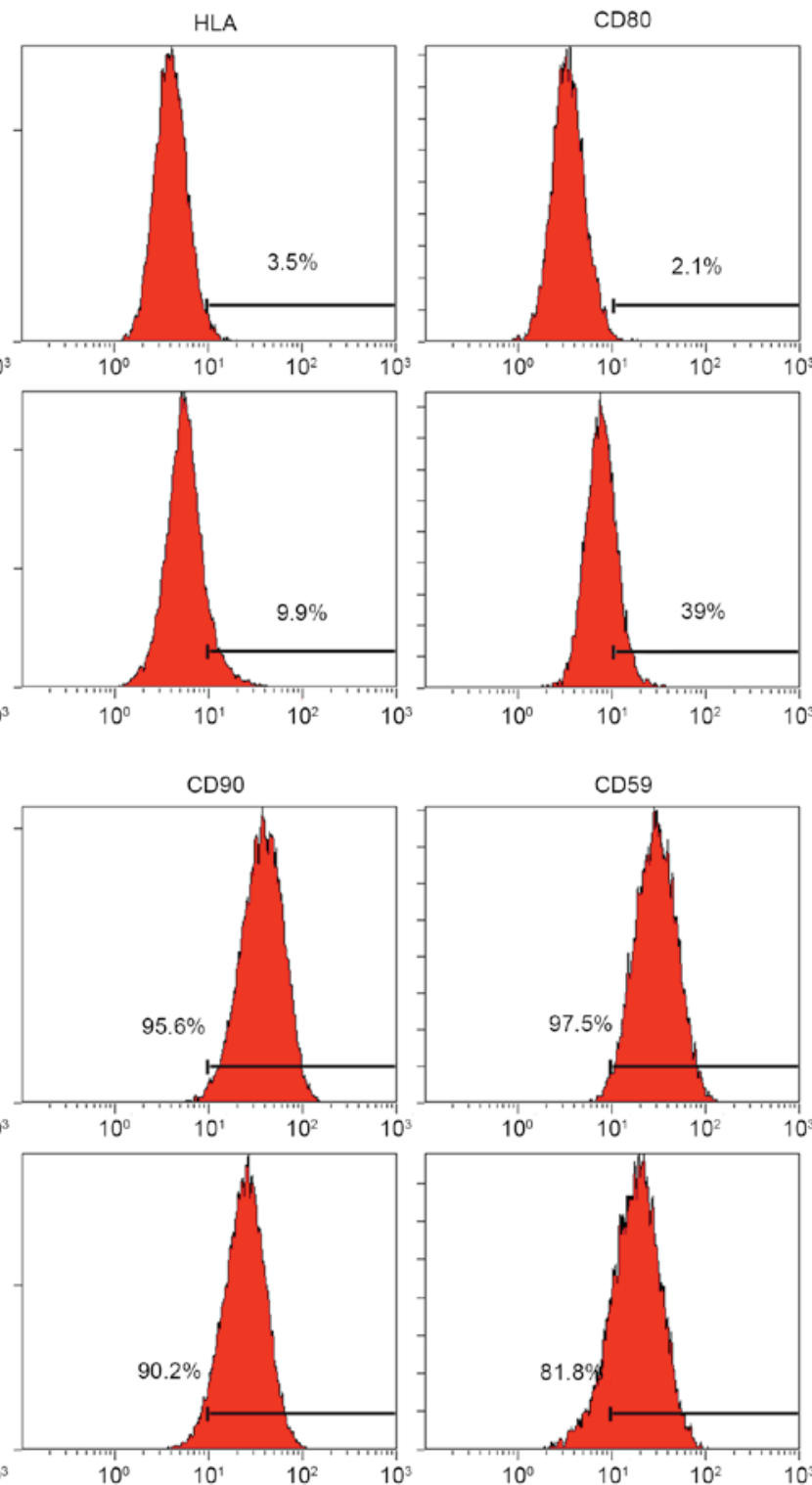

Figure 3. Expression of (A) co-stimulators and (B) surface markers of MSCs. MSCs, mesenchymal stem cells; MSC-TLR8, TLR8 agonist-treated MSCs; TLR-8, Toll-like receptor 8.

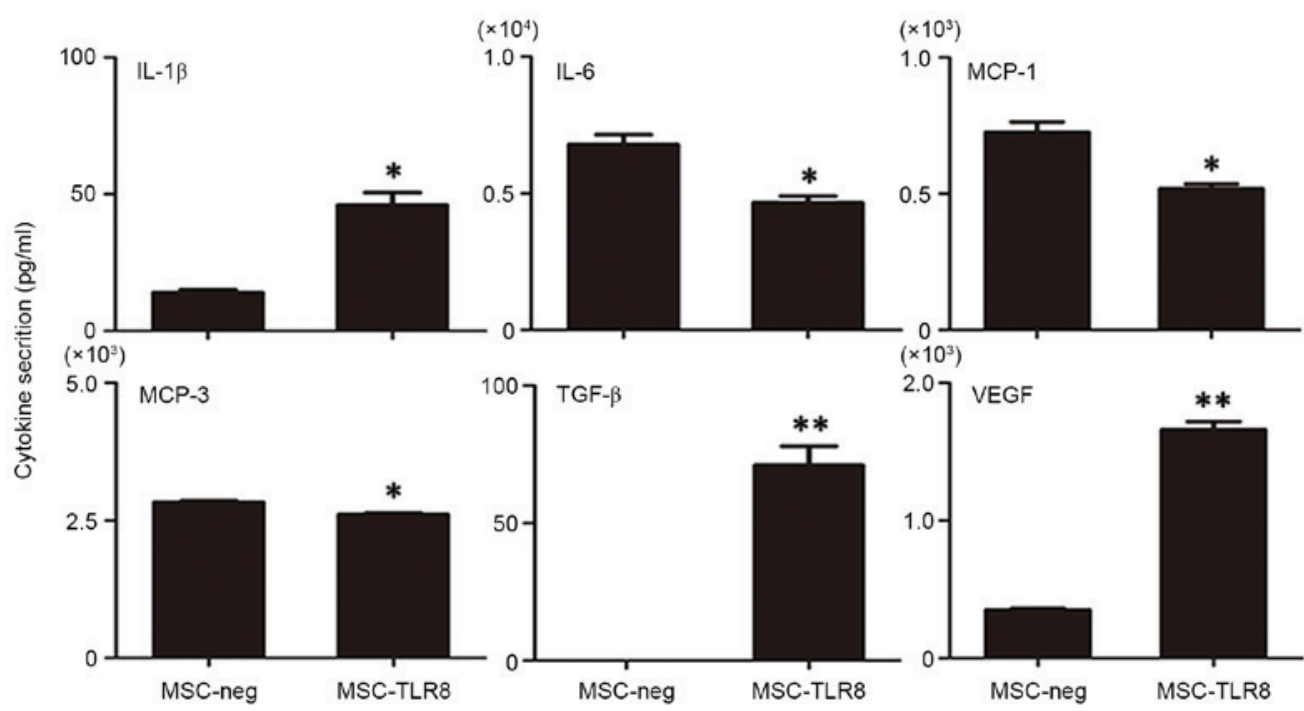

Figure 4. Cytokine/chemokine secretion in culture medium. ${ }^{*} \mathrm{P}<0.05,{ }^{* *} \mathrm{P}<0.001 \mathrm{vs.}$ MSC-neg group. MSCs, mesenchymal stem cells; MSC-TLR8, TLR8 agonist-treated MSCs; TLR-8, Toll-like receptor 8. 
A $5 d$ $10 d$ $15 d$

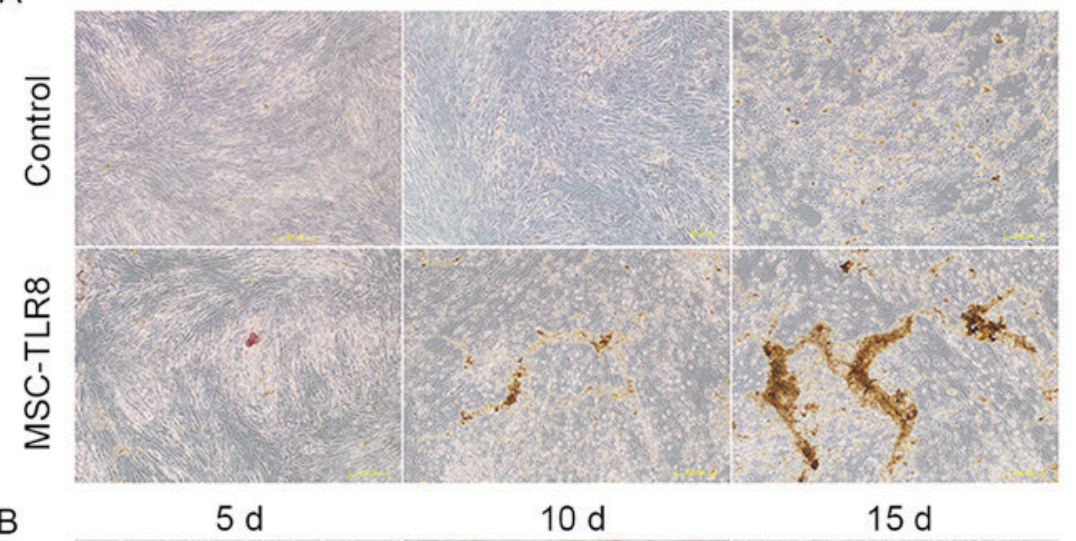

B

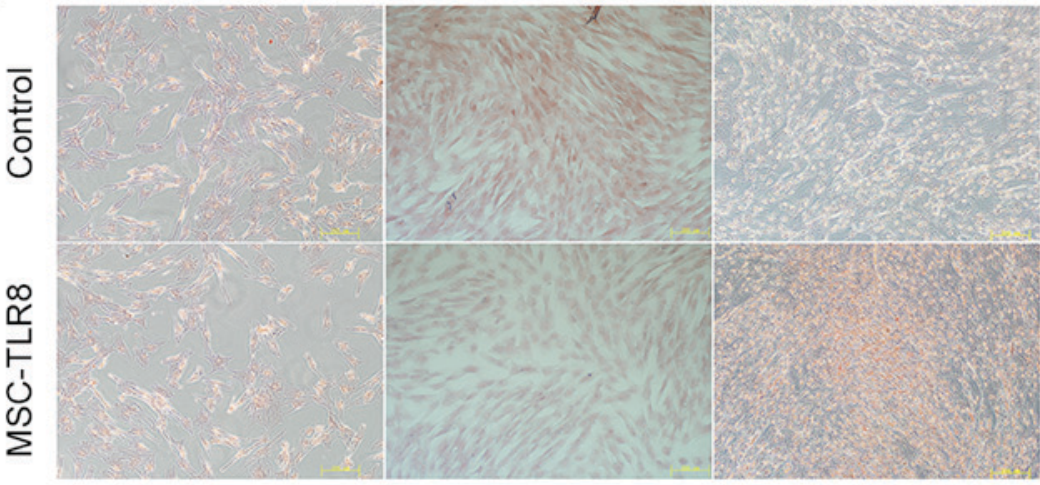

C $5 d$ $10 \mathrm{~d}$ $15 d$

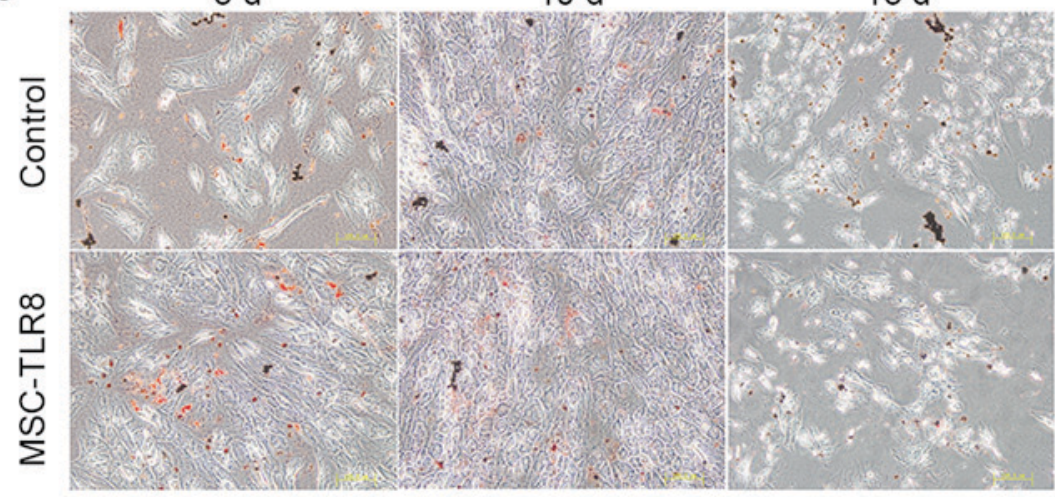

Figure 5. Differentiation of UCMSCs in the presence of TLR8 agonist. (A) Osteocyte differentiation, (B) chondrocyte differentiation and (C) adipocyte differentiation at 5, 10 and 15 days (magnification, x200). Each group is compared with a control group. UBMSC, umbilical cord-derived mesenchymal stem cell; MSC, mesenchymal stem cell; TLR-8, Toll-like receptor 8.

IL-8, MCP-1, MCP-3, MIP-1 $\alpha$, Notch-1, TGF- $\beta$ and VEGF). In these molecules, secretion of TGF- $\beta$ and VEGF was induced markedly upon $\mathrm{R} 848$ stimulation $(\mathrm{P}<0.001)$ and IL-1 $\beta$ also enhanced in the treatment group. The marked decreased secretion in the R848 treatment group was IL-6, MCP-1 and MCP-3 (Fig. 4). Nevertheless, other target genes either did not result in detectable protein levels, or protein levels remained the same. Overall, the secretion pattern of R848-treated UCMSCs appeared to favor pro-inflammatory molecules.

TLR8 enhances the induced osteoblast differentiation of UCMSCs. The effects of TLR8 on the differentiation of UCMSCs into osteocytes, adipocytes and chondrocytes was further examined. UCMSCs were incubated with osteogenic, adipogenic or chondrogenic induction medium, with or without TLR8, and were then allowed to differentiate. As presented in Fig. 5A, the presence of TLR8 in the osteogenic medium greatly enhanced the calcium deposition, which was detected by Alizarin red staining of cells, compared with the control group without R848, especially 10 and 15 days post differentiation. The presence of TLR8 in the adipogenic and chondrogenic medium presented no obvious differences compared with the control groups, and was detected by Oil red O staining and Safranin (Fig. 5B and C).

\section{Discussion}

MSCs are widely believed to have an immunologically privileged phenotype and an immunosuppressive capacity and thus these cells hold potential for clinical applications for minimally invasive cell therapy to promote the regeneration of damaged 
tissue, to treat inflammation and to promote angiogenesis (19). In a series of clinical trials, MSC administration has been demonstrated to be a clinically feasible and relatively safe procedure without major adverse effects in patients with multiple sclerosis, amyotrophic lateral sclerosis and Graft vs. host disease $(9,20)$. However, it is also commonly observed, in human and animal studies, that most of the MSCs disappeared within a few days following infusion, indicating that these cells are rapidly recognized and rejected by the hosts through a yet unknown mechanism (21). The latest disappointing results from MSC clinical trials further support the suspicion that, immediately following infusion, MSC viability and/or activities are markedly reduced, leading to failed treatment efficacy (22).

Toll-like receptors (TLR) were first identified in Drosophila, where they are required both for immunity against pathogens and for embryonic development (23-27). Both TLR7 and TLR8 have been identified as natural receptors for single-stranded RNA, and they are thought to act as potent activators of innate immune responses upon viral infections (28). TLRs activation has been involved in the pathology of a series of inflammatory diseases, as they can either initiate or perpetuate the chronic inflammation attributed to the continue exposure to TLR ligands (29). Therefore, MSCs employed in therapy can be potentially exposed to TLR ligands, which may modulate MSC therapeutic potential in vivo (17). However, there have been no studies about the role of TLR8 in regulating immunogenicity of TLRs in UCMSCs.

In the present study, TLR8was employed to evaluate whether its activation influenced the immunogenicity of USMSCs, including cytokine release, multilineage differentiation and proliferation. In a PBMC-UCMSC co-culture system, it was demonstrated that activation of TLR8 promoted the proliferation of human PBMCs, and the increased release of LDH from R848 stimulation confirmed the occurrence of immune attack; these results clearly demonstrated that activation of TLR8 pathway increased the immune responses. Following this, the expression variation of pro-inflammatory molecules that were important in mediating immune response was measured. The results confirmed the upregulation of many pro-inflammatory cytokines in both mRNA expression and protein secretion, including VEGF, IL6, IL-8, IL-10, CD80 and CD86. Flow cytometry analysis in the present study also confirmed that expression of co-stimulatory factors (CD80, CD86 and HLA-E) in UCMSCs was markedly enhanced in the presence of TLR8 agonist. Huang et al (30) suggested that the enhanced differentiation of MSCs into cardiovascular cells could finally increase the immunogenicity of MSCs. As a result, it was hypothesized that activation of the TLR8 pathway may influence the differentiation ability of UCMSCs and it was demonstrated that the osteoblast differentiation of UCMSCs was significantly enhanced in the presence of the TLR8 agonist. All these observations suggested that TLR8 stimulation in UCMSCs was critical in shaping the immunogenic status of UCMSC.

As a number of endogenous ligands have been identified, including heparin sulfate, hyaluronan, heat shock protein 70 , intracellular components of fragmented cells and eosinophil-derived neurotoxin (14), it was important to confirm the role of TLRs in regulating biological functions, especially immunogenicity of MSC. To the best of the authors' knowledge, the current study was the first report concerning the role of
TLR8 in regulating immunogenicity of UCMSCs. The results clearly indicated that TLR8 activation by agonist has implications for the immune modulating the function of UCMSCs.

The current study provided the foundation for further characterization of TLR8-activated UCMSCs using in vivo experimental systems to establish the physiological role for TLRs in regulation of UCSMC functions.

\section{Acknowledgements}

The present study was supported by a grant from the National Natural Scientific Foundation of China (grant no. 81202023).

\section{References}

1. Colter DC, Sekiya I and Prockop DJ: Identification of a subpopulation of rapidly self-renewing and multipotential adult stem cells in colonies of human marrow stromal cells. Proc Natl Acad Sci USA 98: 7841-7855, 2001.

2. Phinney DG and Prockop DJ: Concise review: Mesenchymal stem/multipotent stromal cells: The state of transdifferentiation and modes of tissue repair-current views. Stem Cells 25: 2896-2902, 2007.

3. Bassi E, Aita CA and Câmara NO: Immune regulatory properties of multipotent mesenchymal stromal cells: Where do we stand? World J Stem Cells 3: 1-8, 2011.

4. Han KH, Ro H, Hong JH, Lee EM, Cho B, Yeom HJ, Kim MG, Oh KH, Ahn C and Yang J: Immunosuppressive mechanisms of embryonic stem cells and mesenchymal stem cells in alloimmune response. Transpl Immunol 25: 7-15, 2011.

5. Van Poll D, Parekkadan B, Cho CH, Berthiaume F, Nahmias Y, Tilles AW and Yarmuch ML: Mesenchymal stem cell-derived molecules directly modulate hepatocellular death and regeneration in vitro and in vivo. Hepatology 47: 1634-1643, 2008.

6. Le Blanc K, Frassoni F, Ball L, Locatelli F, Roelofs H, Lewis I, Lanino E, Sundberg B, Bernardo ME, Remberger M, et al: Mesenchymal stem cells for treatment of steroid-resistant, severe, acute graft-versus-host disease: A phase II study. Lancet 371: 1579-1586, 2008.

7. Vojtassak J, Danisovic L, Kubes M, Bakos D, Jarabek L, Ulicná M and Blasko M: Autologous biograft and mesenchymal stem cells in treatment of the diabetic foot. Neuro Endocrinol Lett 27 (Suppl 2): 134-137, 2006.

8. Ankrum J and Karp JM: Mesenchymal stem cell therapy: Two steps forward, one step back. Tends Mol Med 16: 203-209, 2010

9. Nauta AJ, Westerhuis G, Kruisselbrink AB, Lurvink EG, Willemze R and Fibbe WE: Donor-derived mesenchymal stem cells are immunogenic in an allogeneic host and stimulate donor graft rejection in a nonmyeloablative setting. Blood 108: 2114-2120, 2006.

10. Spaggiari GM, Capobianco A, Becchetti S, Mingari MC and Moretta L: Mesenchymal stem cell-natural killer cell interactions: Evidence that activated NK cells are capable of killing MSCs, whereas MSCs can inhibit IL-2-induced NK-cell proliferation. Blood 107: 1484-1490, 2006.

11. Akira S, Uematsu S and Takeuchi O: Pathogen recognition and innate immunity. Cell 124: 783-801, 2006.

12. Pasare $\mathrm{C}$ and Medzhitov R: Toll-like receptors: Linking innate and adaptive immunity. Adv Exp Med Biol 560: 11-18, 2005.

13. Beutler B, Hoebe K, Du X and Ulevitch RJ: How we detect microbes and respond to them: The Toll-like receptors and their transducers. J Leukoc Biol 74: 479-485, 2004.

14. DelaRosa O and Lombardo E: Modulation of adult mesenchymal stem cells activity by toll-like receptors: Implications on therapeutic potential. Mediators Inflamm 2010: 865601, 2010.

15. Lombardo E, Delarosa O, Mancheño-Corvo P, Menta R, Ramírez C and Büscher D: Toll-like receptor-mediated signaling in human adipose-derived stem cells: Implications for immunogenicity and immunosuppressive potential. Tissue Eng Part A 15: 1579-1589, 2009.

16. Liotta F, Angeli R, Cosmi L, Filì L, Manuelli C, Frosali F, Mazzinghi B, Maggi L, Pasini A, Lisi V, et al: Toll-like receptors 3 and 4 are expressed by human bone marrow-derived mesenchymal stem cells and can inhibit their T-cell modulatory activity by impairing notch signaling. Stem Cells 26: 279-289, 2008. 
17. Tomchuck SL, Zwezdaryk KJ, Coffelt SB, Waterman RS, Danka ES and Scandurro AB: Toll-like receptors on human mesenchymal stem cells drive their migration and immunomodulating responses. Stem Cells 26: 99-107, 2008.

18. Livak KJ and Schmittgen TD: Analysis of relative gene expression data using real-time quantitative PCR and the 2(-Delta Delta C(T)) method. Methods 25: 402-408, 2001.

19. Bianco P, Robey PG and Simmons PJ: Mesenchymal stem cells: Revisiting history, concepts, and assays. Cell Stem Cell 2: 313-319, 2008

20. Karussis D, Karageorgiou C, Vaknin-Dembinsky A, Gowda-Kurkalli B, Gomori JM, Kassis I, Bulte JW, Petrou P, Ben-Hur T, Abramsky O and Slavin S: Safety and immunological effects of mesenchymal stem cell transplantation in patients with multiple sclerosis and amyotrophic lateral sclerosis. Arch Neurol 67: 1187-1194, 2010.

21. Allison M: Genzyme backs Osiris, despite prochymal flop. Nat Biotechnol 27: 966-967, 2009.

22. Li Y and Lin F: Mesenchymal stem cells are injured by complement after their contact with serum. Blood 120: 3436-3443, 2012

23. Hoebe K, Janssen E and Beutler B: The interface between innate and adaptive immunity. Nat Immunol 5: 971-974, 2004.

24. Kambris Z, Hoffmann JA, Imler JL and Capovilla M: Tissue and stage-specific expression of the Tolls in Drosophila embryos. Gene Expr Patterns 2: 311-317, 2002.
25. Beutler B, Jiang Z, Georgel P, Crozat K, Croker B, Rutschmann S, Du X and Hoebe K: Genetic analysis of host resistance: Toll-like receptor signaling and immunity at large. Annu Rev Immunol 24: 353-389, 2006.

26. Ferrandon D, Imler JL and Hoffmann JA: Sensinginfection in Drosophila: Toll and beyond. Semin Immunol 16: 43-53, 2004.

27. Gangloff M, Weber AN, Gibbard RJ and Gay NJ: Evolutionary relationships, but functional differences, between the Drosophila and human Toll-like receptor families. Biochem Soc Trans 31: 659-663, 2003.

28. Schon MP and Schön M: TLR7 and TLR8 as targets in cancer therapy. Oncogene 27: 190-199, 2008.

29. Yamamoto-Furusho JK and Podolsky DK: Innate immunity in inflammatory bowel disease. World J Gastroenterol 13: 5577-5580, 2007.

30. Huang XP, Sun Z, Miyagi Y, McDonald Kinkaid H, Zhang L, Weisel RD and Li RK: Differentiation of allogeneic mesenchymal stem cells induces immunogenicity and limits their long-term benefits for myocardial repair. Circulation 122: 2419-2429, 2010. 